\title{
pXO1-and pXO2-like Plasmids in Bacillus cereus and B. thuringiensis
}

\author{
Seyed Hossein Shahcheraghi ${ }^{1,{ }^{*}}$, Jamshid Ayatollahi ${ }^{1}$ \\ ${ }_{1}^{1}$ Infectious and Tropical Diseases Research Center, Shahid Sadoughi University of Medical Sciences, Yazd, IR Iran \\ ${ }^{*}$ Corresponding author: Seyed Hossein Shahcheraghi, Infectious and Tropical Diseases Research Center, Shahid Sadoughi University of Medical Sciences, Yazd, IR Iran. Tel: +98-913- \\ 2531389, E-mail: shahcheraghih@gmail.com.
}

Received: October 8, 2012; Revised: April 25, 2013; Accepted: April 28, 2013

Keywords: Bacillus cereus; pXO1; pXO2; Plasmids

\section{Dear Editor}

The Bacillus cereus group of organisms contains $B$. thuringiensis, B. anthracis and B. cereus (sensustricto). This group of Gram-positive sporeformers forms a highly homogeneous subdivision of the genus Bacillus. Demonstration of the high genetic relatedness suggests that $B$. anthracis, B. cereus and B. thuringiensis are members of a single species, $B$. cereus sensulato. By definition, $B$. cereus is acrystalliferous, but a $B$. cereus strain carrying a functional cry gene is considered as a $B$. thuringiensis strain. $B$. anthracis is the etiological agent of anthrax, an acute fatal disease found primarily among herbivores, but in fact, all mammals are susceptible. In recent years, it has become best known for its use as a biological weapon (1-3).

In B. anthracis, B. thuringiensis, and the emetic B. cereus, major virulence factors are located extrachromosomally on large plasmids (2). Fully virulent strains of $B$. anthracis carry two large plasmids, pXO1 (181 kb) and pXO2 (96 kb), which encode the machinery necessary to produce and regulate the anthrax virulence factors, the tripartite toxin and the capsule, respectively (1). B. anthracis plasmids can easily be transferred for typing purposes. For example, it is believed that a plasmidless isolates of $B$. anthracis is indistinguishable from $B$. cereus. Numerous studies have attempted to characterize the role of these plasmids in virulence. Generating a plasmid-free strain is important to elucidate the crosstalk between the plasmids and host chromosome $(3,4)$.

Although pXO1 and pXO2 are considered to be specific to $B$. anthracis, there are some reports of rare B. cereus strains harboring plasmids with similarity to these plasmids (4). In Iran, pXO1 plasmid has been transferred from B. anthracis to B. cereus $(5,6)$. A single $208-\mathrm{kb}$ plasmid, named pBc10987, was identified from the non-pathogenic isolate.
B. cereus ATCC 10987.pBc10987 shows surprising similarity to the plasmid pXO1 of B. anthracis (40\% nucleotide identity), however, it lacks the pathogenicity island (PI) containing the genes that encode for the tripartite lethal toxin and its associated regulators. A 191-kb plasmid with a high degree of similarity and synteny to B. anthracis pXO1 was found in B. cereus G9241. In addition, B. cereus G9241 contains a second 218-kb plasmid, previously unidentified, that encodes a novel polysaccharide capsule biosynthetic cluster. These plasmids are expected to contribute greatly to the observed anthrax-like clinical presentation. The pXO1-like plasmid, named pBCXO1, is 99.6\% identical to pXO1 $(7,8)$.

In a study, the plasmids from $B$. cereus isolates that produce emetic toxin or are linked to periodontal disease were sequenced and analyzed. Two periodontal isolates examined contained almost 272-kb plasmids, named pPER272. The emetic toxin-producing isolate contained one $270-\mathrm{kb}$ plasmid, named pCER270, encoding the cereulide biosynthesis gene cluster. Comparative sequence analyses of these B.cereus plasmids revealed a high degree of sequence similarity to the $B$. anthracis pXO1 plasmid, especially in a putative replication region (4).

Recent studies have reported the presence of pXO1-like plasmids in clinical isolates of $B$. cereus, as well as the presence of pXO2-like plasmids in both a clinical strain and a commercial biopesticide strain of B. thuringiensis. B. cereusD-17, B. cereus 43881, and B. thuringiensis 33679 have sequences that are similar to more than one-half of the pXO1 sequences. B. thuringiensis subsp. israelensis carries pBtoxis, and its toxin crystals have been demonstrated to be one of the most toxic combinations tested $(9,10)$. In addition to the toxin genes, pBtoxis encodes a number of genes that are thought to enhance crystal formation and subsequent cell viability by acting as chaperones.

Implication for health policy/practice/research/medical education:

In this brief article, the author summarizes and presents pXO1- and pXO2-like plasmids.These plasmids can be used in producing vaccines against anthrax.

Copyright @ 2013, Ahvaz JundishapurUniversity of Medical Sciences; Published by Kowsar Corp. This is an open-access article distributed under the terms of the Creative Commons Attribution License, which permits unrestricted use, distribution, and reproduction in any medium, provided the original work is properly cited. 
Interestingly, like pBC10987 and pXO1, pBtoxis encodes peptides that are involved in host sporulation and germination (3).

In another study, pAW63 plasmid from the entomopathogenic B. thuringiensis sv. kurstaki was chosen as Gram-positive model plasmid which had homology with several other Gram-positive plasmids most notably with pXO2, the second virulence plasmid of B. anthracis, and pBT9727 was chosen from the pathogenic strain $B$. thuringiensis sv. konkukian 97-27(10).

\section{Financial Disclosure}

There is no financial disclosure.

\section{References}

1. Akhtar P, Khan SA. Two independent replicons can support replication of the anthrax toxin-encoding plasmid pXO1 of Bacillus anthracis. Plasmid. 2012;67(2):111-7.

2. Hoton FM, Andrup L, Swiecicka I, Mahillon J. The cereulide genetic determinants of emetic Bacillus cereus are plasmid-borne. Microbiology. 2005;151(Pt 7):2121-4.

3. Keyhani J, Keyhani E, Attar F, Haddadi A. Sensitivity to detergents and plasmid curing in Enterococcus faecalis. J Ind Microbiol Biotechnol. 2006;33(3):238-42.
4. Rasko DA, Rosovitz MJ, Okstad OA, Fouts DE, Jiang L, Cer RZ, et al. Complete sequence analysis of novel plasmids from emetic and periodontal Bacillus cereus isolates reveals a common evolutionary history among the B. cereus-group plasmids, including Bacillus anthracis pXO1.J Bacteriol. 2007;189(1):52-64.

5. Shahcheraghi SH, Nowruzi J, Javadi G, Shahhoseini MH, Samadi $\mathrm{H}$. [Frequency of pXO1 gene in Bacillus cereus, Bacillus thuringiensis and Bacillus subtilis by SDS-PAGE Technique]. J Sabzevar Univ Med Sci. 2010;17(3):196-206.

6. Shahcheraghi SH, Nowruzi J, Shahhoseini MH, Javadi G, Moradi $\mathrm{H}$. Investigation of pXO plasmid frequency in non-pathogenic Bacilluses by observating plasmid and protein bands in electrophoresis. J Microbiol Biotech. 2010;2(5):41-48.

7. Hoffmaster AR, Ravel J, Rasko DA, Chapman GD, Chute MD, Marston $\mathrm{CK}$, et al. Identification of anthrax toxin genes in a Bacillus cereus associated with an illness resembling inhalation anthrax. Proc Natl Acad Sci US A. 2004;101(22):8449-54.

8. Rasko DA, Ravel J, Okstad OA, Helgason E, Cer RZ, Jiang L, et al. The genome sequence of Bacillus cereus ATCC 10987 reveals metabolic adaptations and a large plasmid related to Bacillus anthracis pXO1. Nucleic Acids Res. 2004;32(3):977-88.

9. Ehling-Schulz M, Fricker M, Grallert H, Rieck P, Wagner M, Scherer S. Cereulide synthetase gene cluster from emetic Bacillus cereus: structure and location on a mega virulence plasmid related to Bacillus anthracis toxin plasmid pXO1. BMC Microbiol. 2006;6:20.

10. Van der Auwera GA, Andrup L, Mahillon J. Conjugative plasmid pAW63 brings new insights into the genesis of the Bacillus an thracis virulence plasmid pXO2 and of the Bacillus thuringiensis plasmid pBT9727. BMC Genomics. 2005;6:103. 